\title{
Surviving acute myocardial infarction: survivin expression in viable cardiomyocytes after infarction
}

\author{
D Santini, A Abbate, S Scarpa, F Vasaturo, G G Biondi-Zoccai, R Bussani, F De Giorgio, F Bassan, \\ D Camilot, M P Di Marino, F Feroce, F Baldi, F Silvestri, F Crea, A Baldi
}

J Clin Pathol 2004;57:1321-1324. doi: 10.1136/icp.2004.018986

See end of article for authors' affiliations

Correspondence to Dr A Baldi, Via G. Orsi, 26-Napoli, Italy; alfonsobaldi@tiscali.it

Accepted for publication 2 June 2004
Background: Apoptosis is a key feature in postinfarction remodelling leading to progressive myocyte loss. Both proapoptotic and antiapoptotic factors contribute to the delicate balance between death and survival. The survivin pathway has emerged as essential in the control of apoptosis, although its role in heart disease is unknown.

Aim: To evaluate survivin expression after acute myocardial infarction (AMI).

Methods: Survivin expression was assessed immunohistochemically in the peri-infarct and remote viable myocardium in 17 consecutive patients who died 1-30 weeks after AMl and in four control hearts.

Results: Survivin was expressed by myocytes in the peri-infarct area in eight patients and in the remote region in 13 patients. The rate of survivin expression after $A M I$ was significantly higher in the remote versus peri-infarct regions and compared with control hearts. Its expression was inversely associated with the presence of dilated cardiopathy and of apoptosis, independently from the gross pathology infarct size. Conclusions: Survivin myocardial expression after AMI may be associated with the survival of at risk myocardium and may be indicative of more favourable remodelling after AMI. These findings identify a potential new target for the treatment of postinfarction remodelling.
A poptosis is a distinct form of cell death that contributes to the active removal of irreversibly damaged or redundant cells in both physiological and pathological processes, such as myocardial ischaemia and infarction. ${ }^{12}$ Recent studies have highlighted the key role of apoptosis in the postinfarction remodelling that leads to progressive myocyte loss in the peri-infarct and remote myocardial regions. ${ }^{2}$ Both proapoptotic and antiapoptotic factors contribute to the delicate balance between programmed cell death and survival. ${ }^{2}$ The survivin pathway has emerged as an essential cellular antiapoptotic mechanism in the control of the upstream initiation of mitochondrial dependent apoptosis. ${ }^{34}$ Survivin is a cytoplasmic protein that exerts an as yet undefined action on mitochondria, leading to resistance to apoptosis. $^{3}$

"The survivin pathway has emerged as an essential cellular antiapoptotic mechanism in the control of the upstream initiation of mitochondrial dependent apoptosis"

Although survivin is not expressed in normal differentiated tissues, it is often highly expressed in cancer, where it behaves also as a promitotic factor controlling chromosome congression and spindle microtubule function. ${ }^{3-5}$ Therefore, survivin is not (or only mildly) expressed in the normal heart, ${ }^{3-5}$ although the role of survivin in acute myocardial infarction (AMI) has received little attention to date. Therefore, the aim of our study was to evaluate survivin expression in the peri-infarct viable myocardium at risk in patients who died 1-30 weeks after AMI, and to correlate survivin expression with signs of unfavourable remodelling, cardiomyocyte apoptosis, and ischaemia.

\section{METHODS}

Survivin expression in cardiomyocytes in the peri-infarct and remote myocardium was assessed by means of immunohistochemistry in 17 consecutive patients selected at postmortem examination who had suffered an AMI 1-30 weeks before death, and who had no clinical or pathological evidence of reinfarction. Four patients with no history of heart disease and a non-cardiac cause of death were selected as controls.

Pathology samples were taken in the peri-infarct and remote viable myocardium. The expression of survivin was assessed by immunohistochemistry performed on $5 \mu \mathrm{m}$ sections of formalin fixed, paraffin wax embedded tissues. Dewaxed sections were first treated in citrate buffer ( $\mathrm{pH}$ 6.0) in a microwave oven at $900 \mathrm{~W}$ for 15 minutes for antigen retrieval, and then immunohistochemistry was performed by a streptavidin-biotin-peroxidase system using a commercial kit (UCS Diagnostic, West Logan, Utah, USA), according to the manufacturer's instructions. Rabbit polyclonal antisurvivin (ABcam, Cambridge, UK) was used as the primary antibody at a 1/1000 dilution. Sections were counterstained with haematoxylin and mounted. Rabbit preimmune serum replaced the primary antibody as a negative control for nonspecific staining. The stained sections were observed using a light microscope and positivity was determined by cytoplasmic staining. Survivin expression was evaluated qualitatively (as positive or negative), and if positive graded on the median number of positive cells in each field and expressed as a percentage (survivin expression index).

Signs of unfavourable cardiac remodelling were sought at necropsy. Eccentric non-compensatory cardiac dilatation was defined as an increased transverse diameter, an increase of the transverse diameter to left ventricle (LV) free wall thickness (greater than 9), and LV wall thickness $\leqslant 11 \mathrm{~mm}$, as described previously. ${ }^{6}$ Infarct size at gross pathology was expressed as per cent of the LV circumference.

Apoptosis in myocytes was assessed by double staining for DNA fragmentation (terminal deoxynucleotidyl transferase mediated dUTP nick end labelling) and activated caspase 3,

Abbreviations: AMl, acute myocardial infarction; COX-2, cyclooxygenase $2 ; \mathrm{HIF}-1 \alpha$, hypoxia inducible factor $1 \alpha ; \mathrm{LV}$, left ventricle 
as described previously. ${ }^{67}$ Because of the potential role of hypoxia in mediating survivin expression, we compared the expression of two hypoxia sensitive markers (hypoxia inducible factor $1 \alpha($ HIF-l $\alpha)$ and cyclooxygenase $2(\text { COX-2) })^{8}{ }^{9}$ with survivin expression. HIF- $1 \alpha$ nuclear expression and COX-2 cytoplasmic expression were assessed by immunohistochemistry, as described previously. ${ }^{8}$ Furthermore, the inflammatory infiltrate was assessed as the number of activated inflammatory cells (expressing the human leucocyte DR antigen) for each unit area using immunofluorescent probes and confocal microscopy, as described previously. ${ }^{10}$

\section{RESULTS}

The median time from AMI to death was 25 days (range, 10$210)$. The median infarct size was $25 \%$. Symptomatic heart failure was present in 10 patients. Trauma was the cause of death in three patients. All patients had at least one significant comorbidity including respiratory failure (nine), gastrointestinal bleeding (seven), sepsis (six), and stroke (four).

Figure 1 shows survivin expression in cardiomyocytes in peri-infarct and remote regions (fig lA, B). The survivin expression index was significantly higher in the remote compared with the peri-infarct regions (median, 10\% (range, $5-25 \%) \quad v 0 \%(0-20 \%) ; p=0.016)$ and compared with control hearts (median, $0 \%(0-2 \%) ; p=0.035$; fig $\mathrm{lC}$ ). Survivin expression was seen in eight of the 17 patients with AMI in the peri-infarct area and in 13 of the 17 patients in the remote region, whereas mild staining only was seen in one of the four control cases $(p=0.081$; fig $1 D)$. Four patients with AMI (24\%) did not express survivin in either the peri-infarct or remote regions.

No significant correlation was found between infarct size and survivin expression. Survivin expression in the periinfarct region was seen less often in patients with eccentric cardiac dilatation $(21 \% \vee 73 \%$ in those without eccentric cardiac dilatation; $p=0.007$ ), and hearts that did not express survivin had greater cardiac diameters than those that did $(\mathrm{p}=0.031$; fig $\mathrm{lD})$. Furthermore, a significant inverse correlation was found between the survivin expression index and the apoptotic rate $(R=-0.40 ; \mathrm{p}=0.020)$, and those hearts lacking survivin expression had higher apoptotic rates both at the infarct site (median, $11.4 \%$ (range, $8.5-14.5 \%) \quad v 2.3 \%(0.5-11.5 \%) ; \mathrm{p}=0.041)$ and in the remote areas (median, $1.3 \%$ (range, $0.4-4.0 \%) v 0.3 \%(0.3-$ $0.5) ; p=0.037$; fig $1 \mathrm{D}$ ). No mitotic features were seen.

Because of the potential role of hypoxia in mediating survivin expression, we correlated the expression of HIF-1 $\alpha$ and COX-2 with that of survivin. HIF- $1 \alpha$ expression was found in nine and COX-2 in six of the 17 myocardial samples. No correlation was found between HIF- $1 \alpha$ or COX-2 and survivin expression. Furthermore, no correlation was found between the number of infiltrating inflammatory cells and survivin expression.

\section{DISCUSSION}

Our study is the first to show myocardial expression of survivin in the human heart after AMI. It also shows that survivin expression is higher in the remote unaffected regions
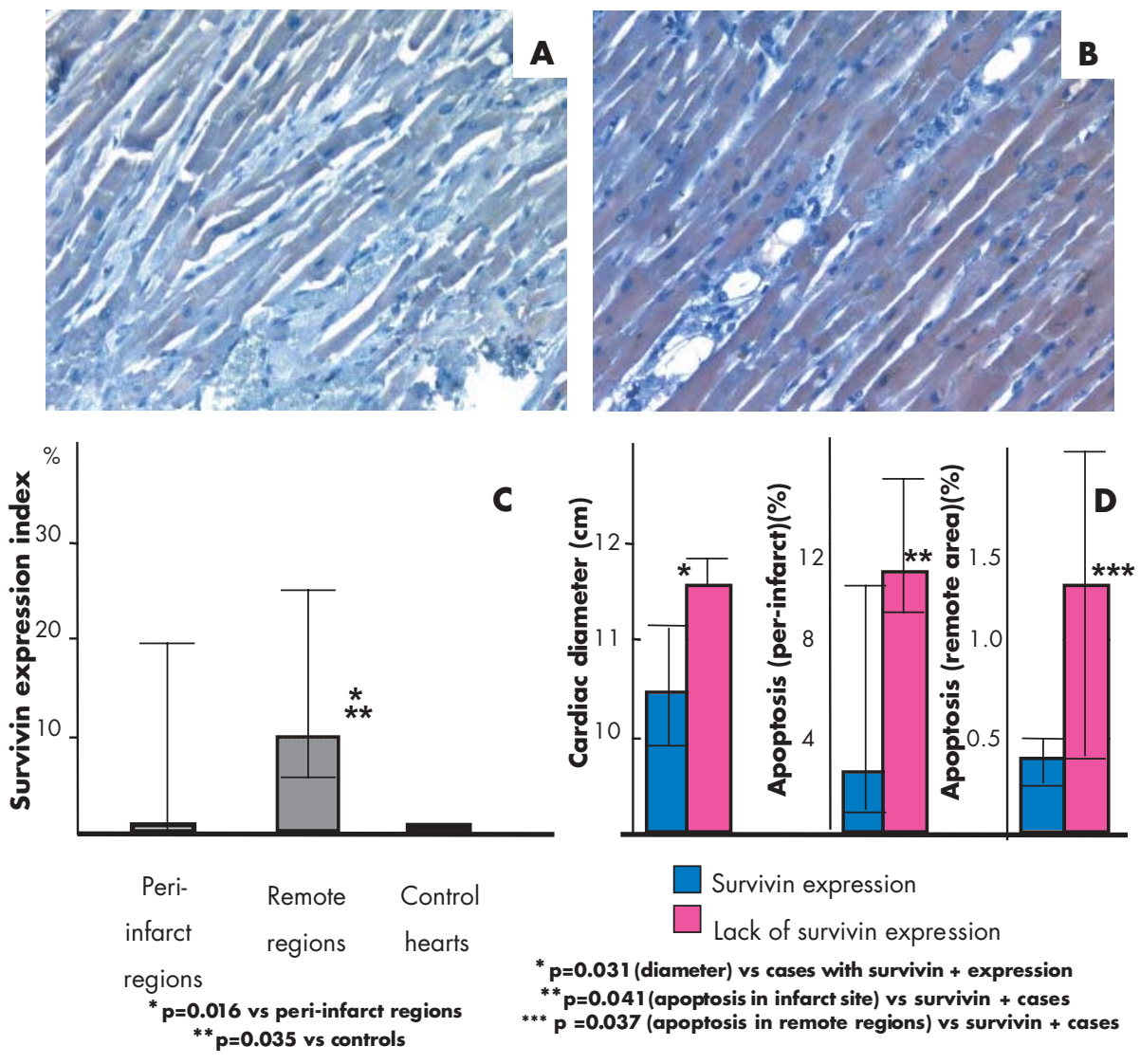

Figure 1 Survivin expression. Cytoplasmic expression was found in (A) peri-infarct and (B) remote myocardium. (C) The survivin expression index was greater in remote compared with peri-infarct regions. (D) Hearts with a lack of survivin expression both in the peri-infarct and the remote regions had greater cardiac diameters and showed higher apoptotic rates, both at the infarct site and in the remote areas, when compared with those that expressed survivin. 
than in the peri-infarct myocardium, and its expression is inversely correlated with the presence of myocyte apoptosis. Furthermore, survivin expression in the peri-infarct myocardium identified a group of subjects with a more favourable remodelling pattern after AMI, suggesting that survivin may be associated with survival of at risk myocardium in patients with AMI. Notably, survivin expression was unrelated to the initial insult, as shown by the lack of correlation between survivin expression and gross pathology infarct size. Although cardiac remodelling is a global process and apoptosis affects both peri-infarct and remote regions, neither apoptosis nor survivin expression is equally distributed, and the greater survivin expression in the remote regions may explain the lower apoptotic rate as a result of the more favourable balance between proapoptotic and antiapoptotic factors.

\section{"Survivin expression in the peri-infarct myocardium identified a group of subjects with a more favourable remodelling pattern after acute myocardial infarction"}

The survivin pathway has recently emerged as a multifaceted and essential cellular mechanism controlling chromosome congression, spindle microtubule fusion, and also the upstream initiation of mitochondrial dependent apoptosis. $^{34}$ This pathway has been mainly investigated in neoplastic disease, in which survivin expression is considered an unfavourable prognostic factor because it provides increased resistance to apoptosis in cancer cells..$^{3-5}$ Therefore, most of the interest in the survivin pathway has been related to the potential to develop treatments that could ultimately counteract survivin expression (that is, protein inhibitors, vaccines) and favourably affect prognosis. Although this may be the case for neoplastic diseases, it may not be so for heart disease. Recent studies pointed out the potential beneficial role of survivin in preventing endothelial apoptosis, thus favouring neoangiogenesis in myocardial ischaemia. ${ }^{11}$ Furthermore, the increased resistance to apoptosis conferred by survivin may be a beneficial physiological response by cardiomyocytes, which face several proapoptotic stimuli. Acute survivin expression has recently been reported in the rim of peri-infarct myocardium surviving the acute stages of AMI (viable myocardium at risk) in an experimental rat model..$^{12}$ Accordingly, several reports (including ours) show that, notwithstanding the significant increase of proapoptotic stimuli in the remote myocardium (that is, ischaemia, increased wall stress, soluble proapoptotic mediators), most cells in both the peri-infarct and remote myocardium retain their viability. Several alterations indicative of the initial stages of apoptosis (such as increased bax to bcl-2 ratios and caspase 3 expression) have been described in hearts with recent AMI, but only a small proportion of cells that are prone to undergo apoptosis show signs of irreversible damage. ${ }^{13} 14$ Thus, a complex network of events affecting the rate of apoptosis and, possibly, stem cell mobilisation, in the weeks and months after the acute coronary event might play a key role in determining the magnitude of cardiac dysfunction and, ultimately, prognosis.

The stimuli that induce survivin expression are still unknown. Because of the potential role of ischaemia in inducing survivin expression, we compared the expression of survivin with two hypoxia sensitive markers (HIF- $1 \alpha$ and COX-2).$^{8}$ ' We were unable to show a significant association between survivin and evidence of tissue ischaemia in our model, suggesting (although not proving) the existence of other stimuli for survivin expression. Moreover, no correlation was found between survivin expression and the number
Take home messages

- We show for the first time that survivin is expressed in the peri-infarct and remote myocardium in human hearts after acute myocardial infarction (AMI)

- This suggests that survivin expression may represent a mechanism by which myocytes at risk of apoptosis retain their viability

- These findings identify a potential new target for the treatment of postinfarction remodelling

of infiltrating inflammatory cells, or with a specific cause of death.

The small sample size and lack of evidence of a causeeffect link between survivin expression and apoptosis, and incomplete assessment of myocyte regeneration are limitations to our present study; however, our data show for the first time that survivin is expressed in the peri-infarct and remote myocardium in human hearts after AMI, suggesting that survivin expression may represent a mechanism by which myocytes at risk of apoptosis retain their viability. Further studies assessing the role of survivin in the heart, the mechanisms of its upregulation, the implications of survivin expression for apoptosis and regeneration of myocytes, and the potential therapeutic enhancement of survivin expression may be of value in preventing postinfarction remodelling and heart failure.

\section{ACKNOWLEDGEMENTS}

This study was supported in part by a FUTURA Onlus grant to DS and $\mathrm{AB}$. The authors wish to thank Dr V Di Trocchio (Richmond, Virginia, USA) for her careful review of the manuscript and illustrations.

\section{Authors' affiliations}

D Santini, Department of Oncology, University Campus Bio-Medico, Rome, 00100 Italy

A Abbate, Department of Internal Medicine, Virginia Commonwealth University, Richmond, VA 23230, USA

G G Biondi-Zoccai, F Crea, Institute of Cardiology, Catholic University, Rome, 00100 Italy

S Scarpa, F Vasaturo, Department of Experimental Medicine and Pathology, University "La Sapienza", Rome, 00100 Italy

R Bussani, F Bassan, D Camilot, F Silvestri, Institute of Pathologic Anatomy, University of Trieste, Trieste, 64100 Italy

F D Giorgio, Department of Forensic Medicine, Catholic University, Rome

A Abbate, Institute of Pathology, University of Torvergata, Rome, 00100 Italy

M P D Marino, F Feroce, F Baldi, A Baldi, Department of Biochemistry, Section of Pathologic Anatomy, Second University of Naples, Naples, 28100 Italy

The first two authors contributed equally to the realisation of this manuscript.

\section{REFERENCES}

1 Krijnen PA, Nijmeijer R, Meijer CJ, et al. Apoptosis in myocardial ischaemia and infarction. J Clin Pathol 2002;55:801-11.

2 Abbate A, Biondi-Zoccai GGL, Baldi A. Pathophysiologic role of myocardial apoptosis in post-infarction left ventricular remodeling. J Cell Physiol 2002; 193: 145-53.

3 Altieri DC. Survivin, versatile modulation of cell division and apoptosis in cancer. Oncogene 2003;22:8581-9.

4 Li F. Survivin study: what is the next wave? J Cell Physiol 2003;197:8-29.

5 Ambrosini G, Adida C, Altieri DC. A novel anti-apoptosis gene, survivin, expressed in cancer and lymphoma. Nat Med 1997;3:917-21.

6 Abbate A, Biondi-Zoccai GGL, Bussani R, et al. Increased myocardial apoptosis in patients with unfavorable left ventricular remodeling and early symptomatic post-infarction heart failure. J Am Coll Cardiol 2003;41:753-60. 
7 Bussani R, Abbate A, Biondi-Zoccai GGL, et al. Right ventricular dilatation after left ventricular acute myocardial infarction is predictive of extremely high peri-infarctual apoptosis at post-mortem examination in humans. J Clin Pathol 2003:56:672-7.

8 Parisi Q, Biondi-Zoccai GGL, Abbate A, et al. Hypoxia-inducible factor-1 expression mediates myocardial response to ischemia late after acute myocardial infarction. Int J Cardiol [ln press.]

9 Abbate A, Santini D, Biondi-Zoccai GGL, et al. Cyclo-oxygenase-2 (COX-2) expression at site of recent myocardial infarction: friend or foe? Heart 2004:90:440-3.

10 Abbate A, Bonanno E, Mauriello A, et al. Widespread myocardial inflammation and infarct-related artery patency. Circulation 2004;110:46-50.
11 Papapetropoulos A, Fulton D, Mahboubi K et al. Angiopoietin-1 inhibits endothelial cell apoptosis via the Akt/survivin pathway. J Biol Chem 2000;275:9102-5.

12 Fukuda S, Kaga S, Sasaki $\mathrm{H}$, et al. Angiogenic signal triggered by ischemic stress induces myocardial repair in rat during chronic infarction. J Mol Cell Cardiol 2004;36:547-59.

13 Scheubel RJ, Bartling B, Simm A, et al. Apoptotic pathway activation from mitochondria and death receptors without caspase-3 cleavage in failing human myocardium: fragile balance of myocyte survival? J Am Coll Cardiol 2002:39:481-8.

14 Baldi A, Abbate A, Bussani R, et al. Apoptosis and post-infarction left ventricular remodeling. J Mol Cell Cardiol 2002;34:165-74.

\section{$\mathrm{ECHO}$}

\section{Commercial chlamydial assay looks promising}

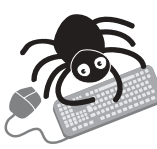

Please visit the Journal of Clinical Pathology website [www. jclinpath.com] for a link to the full text of this article. reliminary results from a Dutch study suggest that a commercial assay for chlamydial heat shock protein 60 (cHSP60) predicts severity of infection in women. At the very least the assay should ensure comparable results among laboratories.

Investigators tested the assay with serum from women well defined for Chlamydia trachomatis infection and disease of the fallopian tubes against results previously obtained with a CT-pELISA IgG assay. Three groups were tested: 21 women confirmed to have no tubal disease (group 1), 86 pregnant women with unknown tubal disease (group 2), and 21 women with confirmed tubal disease (group 3).

The cHSP60 assay performed similarly to the IgG assay, identifying a rising proportion of positives across groups 1-3 (cHSP60: 5\%; 16\%; 27\%; IgG: 19\%; 40\%; 64\%, respectively), which increased for subgroups positive in the IgG assay $(25 \%, 35 \%$, and $43 \%$ across groups $1-3)$, though the trend was not significant. In the IgG negative subgroup, in group 2 , the assay produced only $4 \%$ positives. Median titres in the cHSP60 assay increased across groups $1-3$, as expected for a response linked to disease severity. However, the new assay may identify a different subgroup as it identified only $40 \%$ of women with confirmed tubal disease positive in the IgG assay.

In house cHSP60 assays are widely used to detect $C$ trachomatis infection, and their potential for predicting disease severity has driven the need for a standardised commercial assay. Further testing — of more subjects with different degrees of disease-will be needed to confirm its potential.

\Bax C, et al. Sexually Transmitted Infections 2004;80:415-416. 